\title{
RENORMALIZATION GROUP ANALYSIS FOR THERMAL TURBULENCE
}

\author{
D.N. RIAHI \\ Department of Theoretical and Applied Mechanics \\ 216 Talbot Laboratory, University of Illinois \\ 104 S. Wright St., Urbana, Illinois 61801, U.S.A
}

(Received May 9, 1995)

\begin{abstract}
Renormalization group theory is applied to thermal turbulence. Turbulent fluxes for the flow are accounted for by repeatedly recasting the governing equations with the smallest scales represented by effective larger scales. Expressions for nonlinear contributions to eddy viscosity and eddy diffusivity are determined, and leading order contributions due to buoyancy on various results and equations are estimated. Proper scalings for various quantities and variables including temperature are proposed. The results near two fixed points, which correspond to inertial and buoyancy dominated ranges, are determined and discussed
\end{abstract}

KEY WORDS AND PHRASES: Convection, Turbulence, Thermal, Renormalization, Thermal Turbulence, Turbulent Convection, Renormalization Group, Renormalization Group Analysis

1991 AMS SUBJECT CLASSIFICATION CODES: 76R10, 76D30, 76R99, 80A20, 76E30, 76F10, 76F99.

The present study considers the problem of thermal turbulence modeling analysis. The investigated subject is of tremendous importance in engineering and physical applications, notably in meteorology and oceanography The present modeling analysis is based on the renormalization group (RNG) theory formulated first by Yakhot and Orszag [1] for non-thermal and unbounded flow. Later, Yakhot et al. [2] applied the same theory to non-thermal channel flow and found satisfactory results. Piomelli et al [3] considered an application of RNG to large eddy simulation (LES) of non-thermal boundary layer flow and found reasonably satisfactory results.

Due to large number of scales in fully developed turbulent flow, direct numerical simulation (DNS) is not an efficient procedure to determine solutions of many complex flow problems and LES procedure, where small scales are modeled while large scales are calculated, is preferred. However, previous LES works, which have not been based on RNG modeling, have been shown by Canuto and Minotti [4] and Smith [5] to have serious structural deficiencies and these deficiencies can be amplified for nonequilibrium turbulence problems of technology importance But, recent LES based RNG procedures $[2,3,5]$ led to reasonably satisfactory results and no serious deficiencies were detected. No RNG analysis has yet been done on the present subject, although very recently Riahi [6] applied RNG theory to an incompressible and non-zero-buoyancy flow past an obstacle and estimated the expressions for eddy viscosity and eddy mass diffusivity for the nonlinear dominated range. It is hoped that the results presented in this paper can lead to further progress in thermal turbulence modeling areas These results are applicable both to the so-called inertial range where $-5 / 3$ Kolmogorov law for the energy spectrum 
is still applicable in the presence of buoyancy $[7,8]$ and to the so-called buoyancy range where -3 law for the energy spectrum holds [8]

We consider the thermal turbulent flow which is governed by the continuity, momentum and heat equations which are coupled through the buoyancy force term in the momentum equation Boussinesq approximation together with a linear dependence of density on the temperature $T$ are assumed [7] so that the governing equations can be written in the following form

$$
\begin{gathered}
\frac{\partial \underline{u}}{\partial t}+\underset{u}{u} \cdot \nabla \underline{u}=\frac{1}{\rho_{0}} \nabla p+v_{0} \nabla^{2} \underline{u}+g T \widehat{z} \beta_{0}, \\
\nabla \cdot \underline{u}=0, \\
\frac{\partial T}{\partial t}+\underset{\sim}{u} \cdot \nabla T=\alpha_{0} \nabla^{2} T .
\end{gathered}
$$

Here $u$ is the velocity vector, $p$ is the pressure, $t$ is the time variable, $\rho_{0}$ is a reference (constant) density, $v_{0}$ is the kinetic viscosity, $\beta_{0}$ is the coefficient of thermal expansion, $g$ is the acceleration due to gravity and $\alpha_{0}$ is the thermal diffusivity coefficient Such system of equations can be applied to both open flow systems (such as wake flows) and close flow systems (such as Rayleigh-Benard convection) Following Yakhot and Orszag [1] and their correspondence principle, we add a Gaussian random force $f$ to the momentum equation (1a) so that the resulting system becomes statistically equivalent to the original system (1) at least in the inertial range [8]. We then define the Fourier decomposition of $u, T$ and $p$ fields in the range $k \leq \Lambda$, where $k$ is the magnitude of the wave number vector $\underset{\sim}{k}$ and $\Lambda$ is an ultraviolet cutoff The space-time Fourier-transformed system of governing equations is a system of equations for the

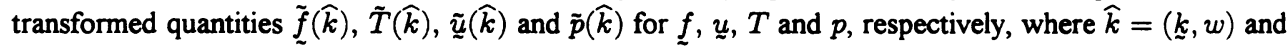
$w$ is the frequency. Next, we assume that the modes vanish for $k$ larger than the ultraviolet cutoff and write

$$
(\tilde{\tilde{f}}, \tilde{\tilde{u}}, \tilde{T}, \tilde{P})=\left(\tilde{f}^{<}, \underline{u}^{<}, T^{<}, p^{<}\right)+\left(\underline{f}^{>}, \underline{u}^{>}, T^{>}, p^{>}\right),
$$

where the quantities with the superscript $<$ (long-wavelength modes) belong to the interval $0<k<\Lambda$ $\exp (-r)$, while the quantities with the superscript $>$ (short-wavelength modes) belong to the interval $\Lambda$ $\exp (-r)<k<\Lambda$. Here $r$ is a positive constant. The expression (2) is used in the governing system for the transformed quantities and two formal parameters $\lambda^{0}(=1)$ and $\delta_{0}(=1)$ are introduced in front of the nonlinear terms in the momentum and heat equations, respectively, to facilitate the perturbation solution of the system.

The RNG procedure consists of two steps. First, all short-wavelength modes terms in the system are removed, up to order $\lambda_{0}^{n} \delta_{0}^{m}(n+m=3)$ and third order or higher of these terms, by repeated substitution of the system for the short-wavelength modes into the system for the long-wave length modes. Second, averages are taken over the part $\underline{f}^{>}$of the random force $\underset{f}{f}$. The following resulting system of equations for $\underline{u}^{<}$and $T^{<}$, which contains only long-wavelength modes terms up to and including order $\lambda_{0}^{n} \delta_{0}^{m}(n+m \leq 2)$, is an approximation which is valid in the limit of small $\widehat{k}$.

$$
\begin{gathered}
\left.-i w+\nu(r) k^{2}\right] u_{l}^{<}(\widehat{k})=f_{l}^{<}(\widehat{k})-i \frac{\lambda_{0}}{2} p_{l m n}(k) \int u_{m}^{<}(\widehat{q}) u_{n}^{<}(\widehat{k}-\widehat{q}) d \widehat{q} /(2 \pi)^{k+1} \\
+\left[\beta_{0} P_{3 l}(\underline{k}) g-\beta_{l}(r) k^{2}\right] T^{<}(\widehat{k}), \\
{\left[-i w+\alpha(r) k^{2}\right] T^{<}(\widehat{k})=-i \delta_{0} k_{l} \int T^{<}(\widehat{q}) u_{l}^{<}(\widehat{k}-\widehat{q}) d \widehat{q} /(2 \pi)^{d+1}+\sigma_{m}(\underline{k}, r) u_{m}^{<}(\widehat{k}),}
\end{gathered}
$$

where $k_{1}, u_{1}$ and $f_{1}$ denote 1-th components of $\underset{k}{k} \underset{\sim}{u}$ and $\underset{f}{f}$, respectively, $i$ is the pure imaginary number, $d$ is the dimension of space, and the expressions for $\nu, \alpha, \beta_{1}, \sigma_{m}, P_{31}$ and $P_{1 m n}$ are given in the appendix. The coefficients $\beta_{1}$ and $\sigma_{m}$ are introduced in (3)-(4) are both due to presence of the buoyancy 
term in the momentum equation, and it is constructive to discuss briefly here the origins of the two terms in (3)-(4) which contain these two coefficients The term $\beta_{1} T^{<}$in (3), which is found to be of order $\lambda_{0} \delta_{0}$, is obtained by first considering the equation for $\underline{u}^{<}$which contains an integral of $\underline{u}^{<} \underline{u}^{>}$Then the equation for $\underline{u}^{>}$, which contains a buoyancy term for $T^{>}$, is used to eliminate $u_{\sim}^{>}$leading to an integral containing $T^{>}$in the equation for $\underline{u}^{<}$. Next, the equation for $T^{>}$, resulted from the heat equation, is used to eliminate $T^{>}$, and lowest order term in the equation for $u^{<}$is applied leading to the term $\beta_{1} T^{<}$The term $\sigma_{m} u_{m}^{<}$in (4), which is found to be the sum of two terms of orders $\lambda_{0} \delta_{0}$ and $\delta_{0}^{2}$, is obtained by first considering the heat equation for $T^{<}$which contains an integral of $T^{<} \underline{u}>$ Then, the equation for $\underline{u}^{>}$, which contains a buoyancy term for $T^{>}$and an integral of $\underline{u}^{<} \underline{u}^{>}$, is used to eliminate $\underline{u}^{>}$Next, the lowest order terms in the equation for $\underline{u}^{<}$plus the heat equation for $T^{>}$are used which lead to the term $\sigma_{m} u_{m}^{<}$

From the results (A1)-(A2) given in the appendix, it is seen that the nonlinear contribution $\nu-\nu_{0}$ to eddy viscosity does not depend on the Prandtl number $P_{0}=\nu_{0} / \alpha_{0}$, while the nonlinear contribution $\alpha-\alpha_{0}$ to eddy diffusivity does depend on $P_{0}$. As can be seen from (3)-(4), some contributions to eddy viscosity and eddy diffusivity come through nonlinear inertial and nonlinear thermal convection terms, while the rest of the contribution come through buoyancy term. The former contribution is essentially the same as in the case of turbulence in the absence of buoyancy [1] and lead to the well known $-5 / 3$ laws for both energy and temperature variance spectrums in the inertial range [1]. Our analysis and results presented above and later will explain why the well known- $5 / 3$ laws still hold for thermal turbulence in the inertial range $[4,7,8]$ (larger $k$ limit range).

Next, following Yakhot and Orszag [1], we rescale the variables and the parameters. Presence of buoyancy and term due to buoyancy in (2)-(3) dictate that rescaling should be according to

$$
\begin{gathered}
k=k^{\prime} \exp (-r),\left(w, \sigma_{m}\right)=\left(w^{\prime}, \sigma_{m}^{\prime}\right) \exp (-a r), \\
\left(\nu, \alpha, \beta_{t}\right)=\left(\nu^{\prime}, \alpha^{\prime}, \beta_{t}^{\prime}\right) \exp [(2-a) r], \\
\left(\underline{u}^{<}, T^{<}\right)=\left(\underline{u}^{\prime<}, T^{\prime<}\right) \exp [(3 a+d+y) r / 2], \\
\underline{f}=\underline{f}^{\prime} \exp [(a+d+y) r / 2],
\end{gathered}
$$

where $a$ is a parameter and $k^{\prime}$ is defined on the same interval $0<k^{\prime}<\Lambda$ as the wavenumber in the original system of equations. Using these rescaling, dropping primes for simplicity of notation and following the same procedure as the one due to ref. 1 for larger $k$ limit range, we find the following results:

$$
\begin{aligned}
& \frac{d \nu}{d r}=\nu\left[a-2+A_{d} \lambda^{2}\right], \\
& \frac{d \alpha}{d r}=\alpha\left[a-2+B_{d} \delta^{2}\right],
\end{aligned}
$$

where these equations are formulated by consideration of asymptotic limit $r \rightarrow 0$ for the larger $k$ limit range [1], and the dimensionless expansion parameters $\lambda$ and $\delta$, introduced in $(6 a, b)$, are satisfied by the following equations

$$
\begin{aligned}
& \frac{2 d \lambda}{d r}=\lambda\left(\epsilon-3 A_{d} \lambda^{2}\right), \\
& \frac{2 d \delta}{d r}=\delta\left(\epsilon-3 B_{d} \lambda^{2}\right),
\end{aligned}
$$

where $\epsilon=4+y-d$ and the expressions for $A_{d}$ and $B_{d}$ are given in the appendix. For $\epsilon<0, \lambda \rightarrow 0$ and $\delta \rightarrow 0$ as $r \rightarrow \infty$. However for $\epsilon>0,(\lambda, \delta)$ approaches a fixed point $\left(\lambda^{*}, \delta^{*}\right)$ as $r \rightarrow \infty$, where

$$
\left(\lambda^{*}, \delta^{*}\right)=\left(\frac{\epsilon}{3}\right)^{1 / 2}\left(A_{d}^{-1 / 2}, B_{d}^{-1 / 2}\right)
$$


At this fixed point, both $\alpha$ and $\nu$ become independent of $r$ if

$$
a=2-\epsilon / 3 \text {. }
$$

Now, in the inertial range (larger $k$ limit range), where the minus five-thirds laws hold for the energy, heat flux and temperature variance spectrums [8], we apply the expressions for these spectrums [8] and find that they hold for $\epsilon=4$ and $a=2 / 3$. Using our above results, it is then seen that $(\nu, \alpha) \sim k^{-4 / 3}$ for the $\epsilon=4$ fixed point.

Before considering the smaller $k$ limit range case, it is useful to modify (3)-(4) to a form where nonlinear and buoyancy contribution to eddy viscosity and eddy diffusivity can be evaluated We find $u_{1}^{<}$ from (3) and use it in (4) and find $T^{<}$from (4) and use it in (3). This procedure leads to a modified form of (3)-(4), where the $\underline{u}^{<}$term in the left hand side of (3), in the limit of $w \rightarrow 0$, becomes

$$
v(r) k^{2} u_{l}^{<}(\widehat{k})+\left\{\left[\beta_{l}(r) k^{2}-\beta_{0} g P_{3 l}(\underline{k})\right] \sigma_{m}(\underline{k}, r) /\left[\alpha(r) k^{2}\right]\right\} u_{m}^{<}(\tilde{k}),
$$

and the $T^{<}$term in the left hand side of (4), in the limit of $w \rightarrow 0$, becomes

$$
\left\{\alpha(r) k^{2}+\left[\beta_{m}(r) k^{2}-\beta_{0} g p_{3 m}(\underset{w}{k})\right] \sigma_{m}(\underset{\sim}{k}, r) /\left[v(r) k^{2}\right]\right\} T^{<}(\tilde{k}) .
$$

It is of interest to note from (10) and (A5)-(A6) in the appendix that for larger $k$ limit range case where $r \rightarrow 0$, buoyancy contributions to (10) are much smaller than the nonlinear contributions to (10) These results provide further supports to our analysis and results for the inertial range presented above Another observation for (3)-(4) and (10) is that, although the nonlinear contributions to eddy quantities does not depend to any particular component of $u$, the buoyancy contributions to eddy quantities does depend, in general, to different components of $\underline{u}$ and provide the effects due to the three components of $\underline{u}$ along any of the coordinate axis.

We now consider the smaller $k$ limit range case. Using the rescaling (5) and formulating in the limit of $k \rightarrow 0$, we find that only part of the buoyancy contributions to (10a) and (10b) designated here by $S_{m}^{(1)}$ and $S_{m}^{(2)}$, respectively, which contain $\tilde{a}_{s m p}$, dominate over the rest of the terms in (10). Investigating either $S_{m}^{(1)}$ or $S_{m}^{(2)}$, we find that either of these quantities satisfy the equation

$$
\frac{d S_{m}^{(\imath)}}{d r}=(\epsilon-6+a) S_{m}^{(2)}, \quad \text { as } \quad r \rightarrow \infty, \quad(i=1,2)
$$

and, thus, $S_{m}^{(\imath)}$ becomes independent of $r$ as $r \rightarrow \infty$, provided that

$$
a=6-\epsilon .
$$

In analogy to the expansion parameters $\lambda$ and $\delta$ and the corresponding equations (7a,b) in the larger $k$ limit range case, new parameters $\lambda_{1}$ and $\delta_{1}$, in the smaller $k$ limit range case, are defined, which satisfy equations of the form

$$
\frac{d}{d r}\left(\lambda_{1}, \delta_{1}\right)=(6-\epsilon)\left(\lambda_{1}, \delta_{1}\right), \quad r \rightarrow \infty .
$$

It is seen from (13) that $\lambda_{1}$ and $\delta_{1}$ become independent of $r$ for $\epsilon=6$ as $r \rightarrow \infty$. Using $\epsilon=6$ in (12), we find $a=0$. It is of interest to note that minus three law $\left(k^{-3}\right)$, that is known to be valid for the energy spectrum in the buoyancy range (smaller $k$ limit range) [8], is followed here for $\epsilon=6$ and $a=0$ As can be seen from (5), $u^{<} \sim T^{<}$so that $k^{-3}$ law follows also for the temperature flux spectrum and for the temperature variance spectrum [8] in the smaller $k$ limit range. Using the above results for the $\epsilon=6$ fixed point and (5), it is then seen that $\left(S_{m}^{(1)}, S_{m}^{(2)}\right) \sim k^{-2}$ for the $\epsilon=6$ fixed point Hence eddy viscosity and eddy diffusivity at the $\epsilon=6$ fixed point dominate those at the $\epsilon=4$ fixed point, determined earlier, for $k \rightarrow 0$ limit, while the opposite is true for the $k \rightarrow \infty$ limit. This result is consistent with the way inertial and buoyancy ranges are defined [8].

The results presented above for both $\epsilon=4$ fixed point (larger $k$ limit range) and $\epsilon=6$ fixed point (smaller $k$ limit range) are in agreement with the physical modelling based on the hypothesis put forward 
by Shur [9] and Lumley [10] about the turbulence spectrum in a free atmosphere according to which the region of the validity of the $k^{-5 / 3}$ law is followed by a region of smaller wave numbers where the $k^{-3}$ law holds

Recently Sukoriansky et al [11] applied the RNG method to study the two dimensionalization of three-dimensional non-thermal turbulence as a result of influence of a strong magnetic field They determined a fixed point corresponding to $\epsilon=6$, where the $k^{-3}$ law was followed by the energy spectrum, and their results were in good agreement with the experimental data due to Sukoriansky and Branover [12] The results of the present investigation, which indicate the preference of the $\epsilon=6$ fixed point and the existence of the $k^{-3}$ law in the buoyancy range of the strong buoyancy dominated turbulence where two-dimensionalization of the flow in the horizontal direction is enhanced, are in agreement with those due to Sukoriansky et al. [11] of their magnetohydrodynamic turbulence studies

An interesting result of the present study was found to be in the buoyancy range $(-3)$ law, which is known to be valid in the stably stratified turbulence in the smaller $k$ limit range, is followed by the energy spectrum $E(k)$ of thermal turbulence, while $E(k)$ follows $(-5 / 3)$ law in the inertia range for the larger $k$ limit range Further support for this result, in addition to those discussed earlier $[10,11]$, can be obtained from very recent DNS studies of turbulent Rayleigh-Benard convection by Kerr [13], which indicate that the mean temperature gradient $S$ of the flow is slightly positive in the region away from the walls, while $S$ is negative near the wall regions. Hence, we may propose the opinion that in thermal turbulence smaller scale eddies in the inertia range favor three-dimensional unstably stratified environment, while larger scale eddies in the buoyancy range favor quasi two-dimensional stably stratified environment.

\section{APPENDIX}

The expressions for $v(r), \alpha(r), P_{31}(\underline{k})$ and $P_{1 m n}(\underline{k})$, introduced in (3)-(4), are given below:

$$
\begin{gathered}
\nu(r)=\nu_{0}+\lambda_{0}^{2} D_{0} S_{d}\left(d^{2}-d-\epsilon\right)[\exp (\epsilon r)-1] /\left[2 \nu_{0}^{2} \Lambda^{\epsilon}(2 \pi)^{d} d(d+2) \epsilon\right], \\
\left.\alpha(r)=\alpha_{0}+\delta_{0}^{2} D_{0}(d-1) S_{d}[\exp (\epsilon r)-1] /\left[\epsilon d \nu_{0}\left(\nu_{0}+\alpha_{0}\right) 2 \pi\right)^{2} \Lambda^{\epsilon}\right], \\
P_{3 l}(\underline{k})=\delta_{3 l}-k_{3} k_{l} / k^{2}, \\
P_{l m n}(\underline{k})=k_{m} P_{\ln }(\underline{k})+k_{n} P_{l m}(\underline{k}),
\end{gathered}
$$

where $D_{0}=T_{0} \nu_{0} K_{B} / \rho_{0}, T_{0}$ is a reference temperature of the fluids, $\rho_{0}$ is a reference fluid density, $K_{B}$ is Boltzmann's constant, $\epsilon=4+y-d, y>-2$ is a constant $(y=-2$ describes fluid in thermal equilibrium, $y>-2$ corresponds to strongly nonequilibrium flow cases [1]), $S_{d}=2 \pi^{d / 2} / \Gamma(d / 2)$ is the area of $d$-dimensional unit sphere [1] and

$$
\delta_{m n}= \begin{cases}1 & \text { for } m=n \\ 0 & \text { otherwise }\end{cases}
$$

The expressions for $\beta_{1}(r)$ and $\sigma_{m}(k, r)$, introduced in (3)-(4) are given below:

$$
\begin{gathered}
2 \beta_{l}(r)=-\lambda_{0} \delta_{0} D_{0} \beta_{0} g\left(d^{2}-2\right) S_{d}[\exp (\widehat{\epsilon} r)-1] \delta_{l 3} /\left[2 \nu_{0}^{2}\left(\nu_{0}+\alpha_{0}\right) d(d+2) \widehat{\epsilon} \Lambda^{\widehat{\epsilon}}(2 \pi)^{d}\right] \\
\widehat{\epsilon} \equiv \epsilon+2, \\
\sigma_{m}(\underline{k}, r)=D_{0} \delta_{0} k_{s}\left([\exp (\epsilon r-4 r)-1]\left\{\lambda_{0} \tilde{a}_{s m p} /\left(\nu_{0} k_{p}\right)\right\} /\right. \\
{\left[2 \beta_{0} g(\epsilon-4)(2 \pi)^{d} \Lambda^{(\epsilon-4)}\right]+[\exp (\epsilon r-2 r)-1] \nu_{0} \delta_{0} k^{2} \widehat{a}_{n s} /} \\
\left.\left\{\left(\alpha_{0}-\nu_{0}\right) k_{n}\left[\beta_{0} g P_{3 m}(\underline{k})-\beta_{m}\right](\epsilon-2) \nu_{0}(2 \pi)^{d} \Lambda^{(\epsilon-2)}\right\}\right)
\end{gathered}
$$


where the coefficients $\widehat{a}_{n s}$ and $\tilde{a}_{s m p}$ are two constants independent of $r$ and $\epsilon$, whose numerical values cannot, in general, be determined analytically, and they are given by the following surface integrals

$$
\begin{gathered}
\widehat{a}_{n s}=\int_{0}^{\pi} d \phi \int_{0}^{2 \pi}\left[p_{3 n}(\underline{q}) p_{k l}(q) / p_{3 l}(q)\right] \sin \theta d \theta\left(q_{k} / q_{s}\right), \\
\tilde{a}_{s m p}=\int_{0}^{\pi} d \phi \int_{0}^{2 \pi}\left\{\left[q_{m} p_{s n}(\underline{q})+q_{n} p_{s m}(\underline{q})\right] p_{n l}(\underline{q}) /\left[p_{3 l}(\underline{q}) q_{p}\right]\right\} \sin \theta d \theta .
\end{gathered}
$$

Here two repeated subscripts mean summation from 1 to 3 and

$$
q_{1} / q=\sin \phi \cos \theta, q_{2} / q=\sin \phi \sin \theta, q_{3} / q=\cos \phi .
$$

Numerical values of $\widehat{a}_{n s}$ and $\tilde{a}_{s m p}$ can, of course, be determined numerically from numerical integrations of the integrals given in (A7)-(A8) but will not be needed here. The expressions for $A_{d}$ and $B_{d}$, introduced in (6)-(7), are given below

$$
\begin{gathered}
A_{d}=S_{d}\left(d^{2}-d-\epsilon\right) /\left[2 d(d+2)(2 \pi)^{d}\right], \\
B_{d}=S_{d}(d-1) /\left[d\left(1+P_{0}\right) P_{0}(2 \pi)^{d}\right] .
\end{gathered}
$$

\section{REFERENCES}

[1] YAKHOT, V. and ORSZAG, S.A., Renormalization group analysis of turbulence, $J$. of Scientific Computing, 1 (1986), 3-51.

[2] YAKHOT, A., ORSZAG, S.A., YAKHOT, V. and ISRAELI, M., Renormalization group formulation of large-eddy simulation, J. of Scientific Computing, 4 (1989), 139-158

[3] PIOMELLI, U, ZANG, T.A., SPEZIALE, C.G. and LUND, T.S., Application of renormalization group theory to the large-eddy simulation of transitional boundary layers, Instablity and Transition, Edited M.Y. Hussaini and R.G. Voigt (Springer-Verlag), II (1990), 480-496

[4] CANUTO, V.M. and MINOTTI, F., Stratified turbulence in the atmosphere and oceans a new subgrid model, J. of Atmospheric Sciences, 50 (1993), 1925-1935.

[5] SMITH, L.M., An adaptation and extension of the Crow constraint for the k-e equations, Proc. of Office of Naval Research Workshop on Non-equilibrium Turbulence (March 1993, Tempe, AZ) (1993), 6-9.

[6] RIAHI, D.N., Renormalization group theory modeling for a stratified turbulence, Proc. of Institute for Computer Applications in Science and Engineering and Langley Research Center Workshop on Transition, Turbulence and Combustion (June-July 1993, Hampton, VA), II (1994), 133-136.

[7] MONIN, A.S. and YAGLOM, A.M., Statistical Fluid Mechanics: Mechanics of Turbulence, 1, MIT Press (Cambridge, MA), 1971.

[8] MONIN, A.S. and YAGLOM, A.M., Statistical Fluid Mechanics: Mechanics of Turbulence, 2, MIT Press (Cambridge, MA), 1975

[9] SHUR, G.N., Experimental investigation of the energy spectrum of atmospheric turbulence, Trudy of Aerological Observation, 43 (1962), 79-90.

[10] LUMLEY, J.L., The spectrum nearly inertial turbulence in a stably stratified fluid, $J$. of Atmospheric Sciences, 21 (1964), 99-102.

[11] SUKORIANSKY, S., STROSELSKY, I., GALPERIN, B, ROY, S.A and ORSZAG, S A., Renormalization group analysis of MHD turbulence with low magnetic Reynolds numbers, Progress in Astronautics and Aeronautics, 116 (1992), 151-158.

[12] SUKORIANSKY, S. and BRANOVER, H., Turbulence peculiarities caused by interference of magnetic fields with the energy transfer henomena, Progress in Astronautics and Aeronautics, 112 (1988), 87-99.

[13] KERR, R.M., Rayleigh number scaling in numerical convection, to appear in J. of Fluid Mechanics (1995). 


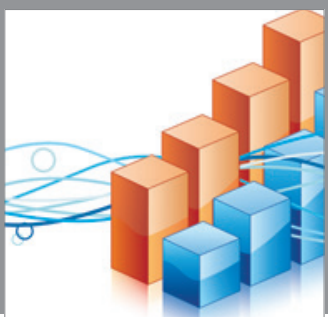

Advances in

Operations Research

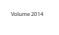

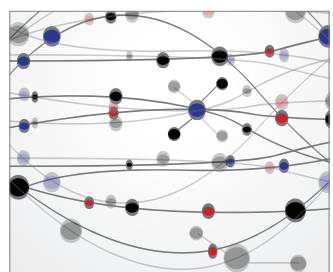

\section{The Scientific} World Journal
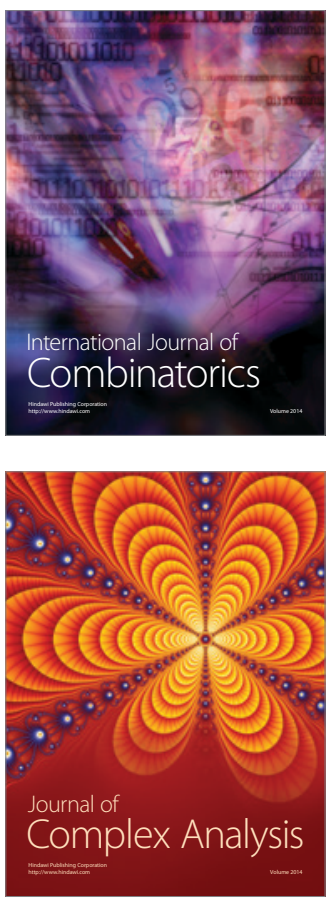

International Journal of

Mathematics and

Mathematical

Sciences
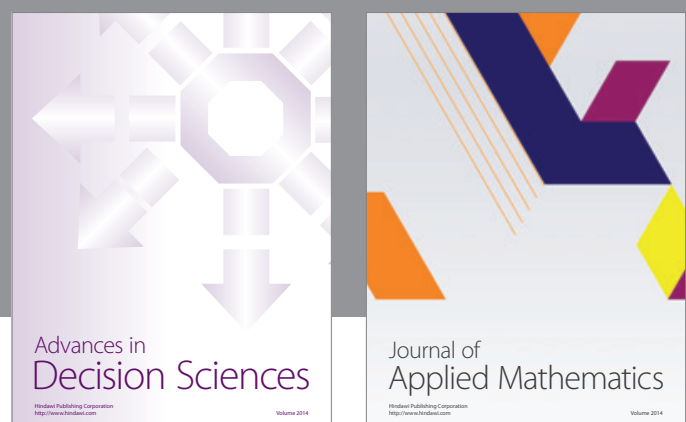

Journal of

Applied Mathematics
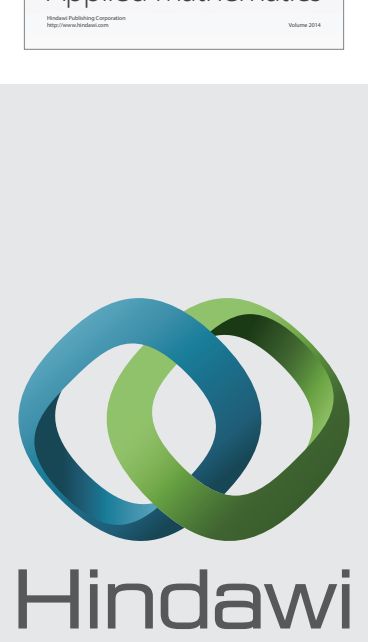

Submit your manuscripts at http://www.hindawi.com
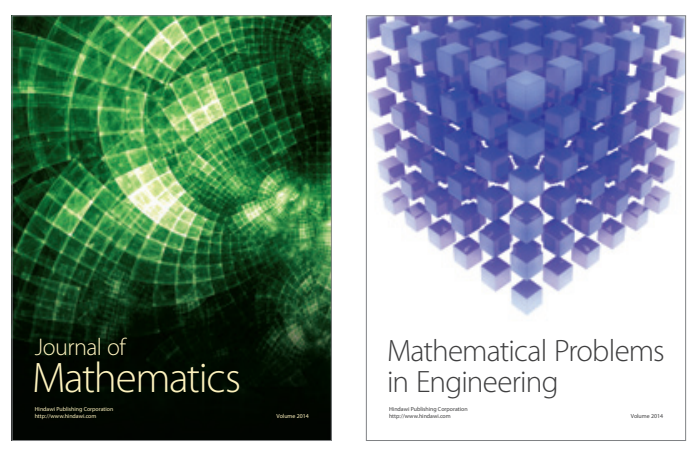

Mathematical Problems in Engineering
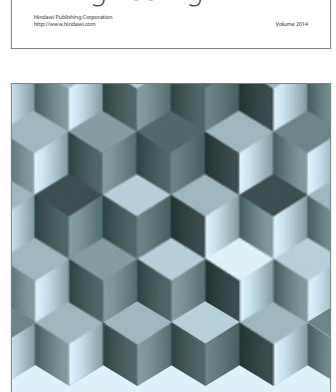

Journal of

Function Spaces
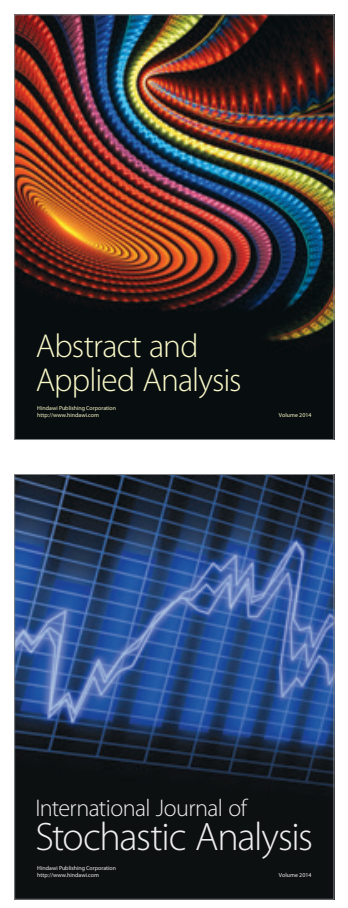

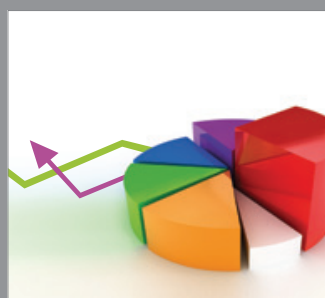

ournal of

Probability and Statistics

Promensencen
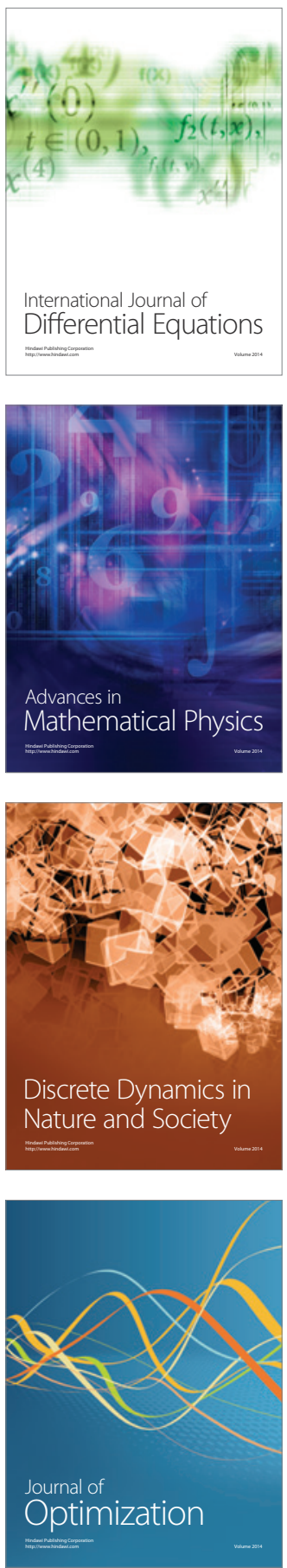